\title{
Do we need to treat all newborns borm to mothers with suspected chorioamnionitis?
}

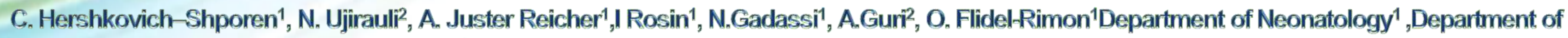
Pediatrics ${ }^{2}$, Kaplan medical center, Rehovot, Hebrew university, Jerusalem' Israel.

\section{Background}

In 2015, we changed the protocol for early detection and treatment of early onset sepsis (EOS) based on the 2010 recommendations of the Centers for Disease Control and Prevention (CDC). The new indications for antibiotic therapy were symptomatic newborns suspected for EOS or newborns born to mothers with suspected chorioamnionitis.

In this study, we prospectively evaluated the new protocol and evaluated the need to treat all newborns born to mothers with suspected chorioamnionitis.

Methods - Inclusion criteria

Gestational age of 35 weeks or more.

Symptomatic newborns and/or newborns receiving antibiotic treatment during the first 72 hours after delivery.

Newborns whose mothers were treated with intra partum antibiotic therapy (IAT) because of the following risk factors for EOS: maternal Group B streptococcus (GBS) carrier state, maternal fever $38^{\circ} \mathrm{C}$ and above during labor, preterm labor, prolonged premature rupture of membranes (PPROM), and suspected chorioamnionitis.

Newborns with proven sepsis.

Study design

We prospectively followed for one year (May 2015 - April 2016) all newborns that met the inclusion criteria. Data was collected regarding baseline maternal, perinatal and neonatal courses together with details of the sepsis events from the medical records.

Study population

A total of 7062 newborns were born at KMC during the study period. Out of them, 1345 (19\%) newborns met study criteria.

Mean birth weight was $3184 \pm 486$ grams (range $1985-5255$ grams) and mean gestational age was 38 \pm 1.7 weeks (range $35-42)$. Twohundred and four newborns (14\%) were given antibiotic treatment. Six newborns had proven EOS, all of them were symptomatic. Two of them also had suspected

maternal chorioamnionitis. No asymptomatic newborn had proven sepsis (including newborns with suspected maternal chorioamnionitis).

Table 1 Maternal risk factors

\begin{tabular}{|l|c|l|}
\hline Included Total & 1345 & \\
\hline $\begin{array}{l}\text { Maternal risk factors } \\
\text { (some had more than } \\
\text { one risk factor) }\end{array}$ & 1294 & $\begin{array}{l}\text { GBS carrier - 625 } \\
\text { Preterm labor - 190 } \\
\text { PROM - 287 } \\
\text { Maternal fever - 137 } \\
\text { Chorioamnionitis - 89 }\end{array}$ \\
\hline $\begin{array}{l}\text { Maternal intrapartum } \\
\text { antibiotic prophylaxis } \\
\text { (IAP) }\end{array}$ & 1069 & \\
\hline
\end{tabular}

Table 2 Neonatal antibiotic treatment and sepsis

\begin{tabular}{|l|c|c|}
\hline Included & \multicolumn{2}{|c|}{$\mathbf{1 3 4 5}$ neonates } \\
\hline Neonatal Antibiotic therapy & 204 & Sepsis 6 \\
\hline symptomatic & 123 & Sepsis 6 \\
\hline Chorioamnionitis & 89 & Sepsis 2 \\
\hline $\begin{array}{l}\text { Both (symptoms and } \\
\text { chorioamnionitis) }\end{array}$ & 14 & Sepsis 2 \\
\hline Antibiotic treatment for other reasons & 6 & Sepsis 0 \\
\hline
\end{tabular}

Results

Of the included neonates, $89 / 1345$ (6.62\%) had maternal suspected chorioamnionitis, two (2.25\%) had proven EOS, but were also symptomatic. The RR for symptomatic newborn to have EOS was $10.9(\mathrm{Cl} 3.5-33.39, \mathrm{P}<0.0001)$ and the NNT was 22.6 , while the RR for newborn with suspected maternal chorioamnionitis was $5.03(\mathrm{Cl} 1.03-24.6 \mathrm{P}=0.045)$ and the NNT was 55.5. Symptomatic newborns were at 2.2 increased risks to have EOS compared with newborns with suspected maternal chorioamnionitis.

\section{Conclusions}

The current protocol (CDC2010) was found to be effective and safe in diagnosing and treating all cases of EOS. All newborns with proven sepsis were symptomatic. According to the protocol, we also treated 75 asymptomatic newborns whose mothers had suspected choroiamnionis.

We suggest that asymptomatic newborns born to mothers with suspected chorioamnionitis should not be automatically treated with antibiotics. However, more studies are needed to confirm the above data. 